\title{
The Role of Serum Inflammation-Based Factors in Anti-Vascular Endothelial Growth Factor Treatment for Macular Edema Secondary to Retinal Vein Occlusion and Its Subtypes
}

\author{
Jie Rao ${ }^{a} \quad \mathrm{Na} \mathrm{Wu}^{\mathrm{a}}$ Xiaoyong $\mathrm{Qu}^{\mathrm{b}}$ Yuxiang $\mathrm{Hu}^{\mathrm{a}}$ Yajun $\mathrm{Wu}^{\mathrm{a}} \quad \mathrm{Yi}_{\mathrm{C}}$ Cheng ${ }^{\mathrm{a}}$ \\ Li Yan $^{\mathrm{a}}$ Hui Huang ${ }^{\mathrm{a}}$ Siyu $\mathrm{Li}^{\mathrm{a}}$ Ling Shi $^{\mathrm{a}}$ Xiaorong $\mathrm{Wu}^{\mathrm{a}}$ \\ a Department of Ophthalmology, the First Affiliated Hospital of Nanchang University, Nanchang, China; \\ ${ }^{b}$ Department of Ophthalmology, Jiuiang No. 1 People's Hospital, Jiujiang, China
}

\section{Keywords}

Retinal vein occlusion - Macular edema ·

Platelet-to-lymphocyte ratio - Anti-vascular

endothelial growth factor treatment

\begin{abstract}
Objective: The aim of this work was to evaluate the association between pretreatment inflammation-based factors and outcomes in patients with macular edema (ME) secondary to retinal vein occlusion (RVO) and its subtypes after intravitreal ranibizumab or conbercept implant. Methods: This retrospective observational study included patients who were diagnosed with ME secondary to RVO at the First Affiliated Hospital of Nanchang University between January 2017 and January 2019, and who subsequently received intravitreal anti-vascular endothelial growth factor (VEGF) treatment. Blood-based parameters were measured before treatment, and correlations between best-corrected visual acuity (BCVA) and each of 3 parameters - neutrophil-to-lymphocyte ratio (NLR), monocyte-to-lymphocyte ratio (MLR), and platelet-to-lymphocyte ratio (PLR) - were analyzed to identify predictors of effective intravitreal injection treatment
\end{abstract}

outcomes. Results: A total of 315 treatment-naïve eyes treated with anti-VEGF drugs for RVO-ME were retrospectively analyzed in this study. The mean PLR value was significantly different in the effective and ineffective group for RVO-ME (138.03 \pm 48.61 vs. $106.79 \pm 27.28)$, branch RVO (BRVO)-ME (216.47 \pm 53.04 vs. $185.94 \pm 51.47$ ), and central RVO (CRVO)ME $(231.07 \pm 66.05$ vs. $196.20 \pm 60.44)$. The cutoff value of the PLR was 97.92, the area under the curve was 0.70 , and the sensitivity and specificity were 81.5 and $44.3 \%$, respectively. The mean NLR value was significantly different in the effective and ineffective groups for RVO-ME ( $2.20 \pm 1.40$ vs. 1.92 $\pm 0.89)$, and BRVO-ME ( $2.01 \pm 0.80$ vs. $1.82 \pm 0.84)$, but not in patients with CRVO-ME $(2.51 \pm 2.02$ vs. $2.12 \pm 0.95)$. There are no significant differences between BRVO-ME and its subtype groups in MLR values. But the mean MLR value was significantly higher in the conbercept group than in the ranibizumab group among patients in the effective group $(0.27 \pm$ 0.11 vs. $0.25 \pm 0.14$ ). Conclusion: Higher pretreatment PLR was associated with BCVA in patients with RVO-ME and its subtypes who were treated with anti-VEGF drugs. The PLR may be used as a predictive and prognostic tool for effective intravitreal injection treatment outcomes.

(C) 2020 S. Karger AG, Basel karger@karger.com

www.karger.com/ore

Karger $\stackrel{2}{*}$ (c) 2020 S. Karger AG, Base

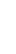

Xiaorong $\mathrm{Wu}$

Department of Ophthalmology

The First Affiliated Hospital of Nanchang University

Nanchang 330006 (China)

wxr98021@126.com 


\section{Introduction}

Retinal vein occlusion (RVO) is the second most common retinal vasculopathy after diabetic retinopathy. Depending on the location of obstruction, RVO can be differentiated into central RVO (CRVO) and branch RVO (BRVO) [1]. A multicenter study showed that the prevalence rates for BRVO and CRVO were 4.42 and 0.80 per 1,000 persons; these varied among ethnic groups and increased with age, but were not affected by sex $[1,2]$. Vascular occlusion leads to retinal ischemia, which can increase vascular permeability and cause the accumulation of secondary intraretinal fluid and subretinal fluid in the outer plexiform layer and subretinal space. Macular edema (ME) easily occurs secondary to these pathophysiologic changes, typically producing secondary retinal pigment epithelial changes that lead to poor visual acuity.

Risk factors of RVO are known to include hypertension, systemic vascular disease, hyperlipidemia, pregnan$c y$, oral contraceptive use, and hypercoagulability; however, the underlying pathogenesis of RVO remains unclear [3]. Interestingly, some studies have shown that local and systemic inflammation can induce both atherosclerosis and systemic hypercoagulability, which influence the development of ME secondary to RVO (RVOME) [4]. Several studies have shown that the levels of inflammatory factors (e.g., vascular endothelial growth factor [VEGF], interleukin-1 $\beta$, and interleukin-6) are associated with the severity of ME [5]. In addition, aqueous humor levels of proinflammatory cytokines are reduced after dexamethasone treatment in patients with RVO [6].

VEGF is an endothelial cell mitogen and vasopermeability factor that can cause conformational phosphorylation and changes in protein content in the tight junctions of retinal vascular endothelial cells [7]. The relatively recent introduction of anti-VEGF drugs has aided in the treatment of many retinal diseases, including age-related macular degeneration, radiation retinopathy, and diabetic ME, especially when pathological changes involve ME and neovascularization. In RVOs, elevated secretion of VEGF leads to elevated vascular permeability and vasodilation, which aggravates the severity of ME [8]. Thus, VEGF inhibitors have become the primary treatment approach for RVO-ME, which has significantly impacted the treatment and visual outcomes in RVO-ME [9]. In the past decade, clinical trials have increasingly shown that, compared with macular grid laser and panretinal photocoagulation, anti-VEGF therapy (e.g., intravitreal injections of ranibizumab [IVR] or conbercept [IVC]) could bring superior visual acuity [9].
There is growing evidence to support the relationship between the inflammatory response and RVO-ME [5]. Serum inflammation-based markers (e.g., neutrophil-tolymphocyte ratio [NLR], monocyte-to-lymphocyte ratio [MLR], and platelet-to-lymphocyte ratio [PLR]) reportedly can be used as prognostic indicators for many conditions, including cancers [10], cardiovascular diseases [11], and inflammatory or infectious pathologies [12]. Recently, the level of serum NLR ratio was reported to be correlated with RVO [13]. However, the relationships between serum inflammation-based markers and RVO$\mathrm{ME}$, especially in patients receiving anti-VEGF therapy, have not been studied in detail. Therefore, this study was performed to investigate levels of serum inflammationbased factors and explore their relationship with effectiveness in patients with RVO-ME who were treated with ranibizumab or conbercept.

\section{Materials and Methods}

This retrospective study included 315 patients who were diagnosed with RVO-ME and received initial anti-VEGF therapy between January 2017 and January 2019 at the First Affiliated Hospital of Nanchang University. All patients underwent a complete ocular examination including best-corrected visual acuity (BCVA), slit-lamp biomicroscopy, and fundus biomicroscopy. Based on an ophthalmoscopic fundus examination, patients who had hemorrhages, cotton wool-spots, edema, and venous dilatation and tortuosity originating from arteriovenous crossing in a localized retinal area were diagnosed with BRVO, and patients who had pathologic changes in 4 retina quadrants accompanied by retinal vein dilatation were diagnosed with CRVO. In accordance with the American Academy of Ophthalmology guidelines, RVO-ME was detected using optical coherence tomography (Cirrus HD - OCT; Carl Zeiss Meditec Inc., Dublin, CA, USA) and fundus fluorescein angiography. All optical coherence tomography exams were performed by the same qualified physician who was blinded to the patients' vision parameters when analyzing the optical coherence tomography images.

Patients were excluded if they met any of the following conditions: (1) other treatments for RVO during the follow-up period, such as laser photocoagulation, intraocular steroid treatment, or periocular steroid treatment; (2) other concomitant ocular diseases such as posterior uveitis or age-related macular degeneration; (3) history of an acute coronary event or stroke in the previous 3 months; (4) presence of glaucoma, diabetes, systemic inflammatory diseases, cardiovascular, hepatic, and renal disorders, blood dyscrasias, and/or malignancy; (5) history of routine anti-inflammatory drug, oral contraceptive, and/or anticoagulants use; (6) history of smoking and/or alcohol consumption; (7) occurrence of vitreous hemorrhage and infection after surgery.

The specific anti-VEGF drug (ranibizumab or conbercept) used for therapy in this study was determined by the treating physician and patients. All included patients received intravitreal injections of $0.5 \mathrm{mg} / 0.05 \mathrm{~mL}$ ranibizumab or conbercept under asep- 
Table 1. Comparison of the ineffective group and the effective group with RVO-ME

\begin{tabular}{|c|c|c|c|}
\hline & $\begin{array}{l}\text { Ineffective group } \\
(n=158)\end{array}$ & $\begin{array}{l}\text { Effective group } \\
(n=157)\end{array}$ & $p$ value \\
\hline \multicolumn{4}{|l|}{ Baseline characteristics } \\
\hline Age, years & $57.34 \pm 11.88$ & $58.90 \pm 12.20$ & 0.18 \\
\hline Male/female & $79 / 79$ & $76 / 81$ & 0.78 \\
\hline BRVO/CRVO & $103 / 55$ & $99 / 58$ & 0.69 \\
\hline Ranibizumab/conbercept & $105 / 53$ & $100 / 57$ & 0.61 \\
\hline Preoperative BCVA, logMAR & $0.75 \pm 0.40$ & $0.79 \pm 0.40$ & 0.41 \\
\hline Postoperative BCVA, logMAR & $0.78 \pm 0.42$ & $0.55 \pm 0.32$ & $<0.01$ \\
\hline Changes in BCVA, logMAR & $0.02 \pm 0.08$ & $0.26 \pm 0.19$ & $<0.01$ \\
\hline Preoperative IOP, mm Hg & $14.11 \pm 2.60$ & $14.04 \pm 2.91$ & 0.87 \\
\hline Postoperative IOP, $\mathrm{mm} \mathrm{Hg}$ & $13.82 \pm 2.69$ & $13.72 \pm 2.70$ & 0.78 \\
\hline Changes in IOP, $\mathrm{mm} \mathrm{Hg}$ & $2.47 \pm 1.68$ & $2.13 \pm 1.47$ & 0.09 \\
\hline \multicolumn{4}{|l|}{ Laboratory findings } \\
\hline White blood cells, $\times 10^{9} / \mathrm{L}$ & $5.79 \pm 1.95$ & $5.72 \pm 1.50$ & 0.97 \\
\hline Neutrophils, $\times 10^{9} / \mathrm{L}$ & $3.35 \pm 1.54$ & $3.42 \pm 1.18$ & 0.21 \\
\hline Monocytes, $\times 10^{9} / \mathrm{L}$ & $0.42 \pm 0.18$ & $0.41 \pm 0.15$ & 0.40 \\
\hline Platelets, $\times 10^{9} / \mathrm{L}$ & $189.51 \pm 54.78$ & $221.87 \pm 58.59$ & $<0.01$ \\
\hline Lymphocytes, $\times 10^{9} / \mathrm{L}$ & $1.83 \pm 0.54$ & $1.73 \pm 0.58$ & 0.06 \\
\hline NLR & $1.92 \pm 0.89$ & $2.20 \pm 1.40$ & 0.01 \\
\hline MLR & $0.24 \pm 0.11$ & $0.26 \pm 0.13$ & 0.21 \\
\hline PLR & $106.79 \pm 27.28$ & $138.03 \pm 48.61$ & $<0.01$ \\
\hline
\end{tabular}

RVO, retinal vein occlusion; ME, macular edema; BRVO, branch RVO; CRVO, central RVO; BCVA, bestcorrected visual acuity; IOP, intraocular pressure; NLR, neutrophil-to-lymphocyte ratio; MLR, monocyte-tolymphocyte ratio; PLR, platelet-to-lymphocyte ratio.

tic conditions. If the postoperative BCVA improved compared with preoperative $\mathrm{BCVA}$, we considered the treatment effective; otherwise, we considered it to be ineffective. In patients who received treatment in both eyes, only the eye that first received treatment was analyzed. If patients had accepted more than one treatment, we just analyzed the first intervention. All patients provided written consent for IVR or IVC treatment and for the use of their data for research purposes. Patients returned for follow-up 1 week postoperatively, then were evaluated monthly and received injections as needed or pro re nata.

Patients' peripheral blood samples for complete blood counts were obtained between 5:00 and 6:00 a.m. from the antecubital vein before IVR or IVC treatment. Leukocyte subtypes were measured using an automated blood coagulation analyzer (XE-2100; Sysmex, Kobe, Japan). NLR, MLR, and PLR were calculated and recorded for all blood samples.

\section{Statistical Analysis}

All statistical analyses were performed using SPSS 19.0 software (SPSS Inc., Chicago, IL, USA). Parametric data are reported as the mean \pm SD and categorical data as percentages. Independent parameters were compared via the independent-sample $t$ test or using the Mann-Whitney $U$ test if there was no normal distribution. The $\chi^{2}$ test was used to evaluate the categorical data and determine associations between dichotomous variables. The correlation was evaluated by Spearman correlation test. The optimal cutoff point of NLR, PLR, and MLR was determined by receiver operator characteristic (ROC) curve analysis. The areas under the curve (AUC) were calculated to analyze the accuracy of the tests. A $p$ value $<0.05$ was significant in all of the analyses.

\section{Results}

A total of 315 treated eyes of 315 patients diagnosed with any type of RVO-ME were included in this study; all patients had received intravitreal injections of anti-VEGF drugs. The mean age of the ineffective and effective groups were $57.34 \pm 11.88$ and $58.90 \pm 12.2$ years, respectively. The clinical features and laboratory characteristics of the patients are summarized in Table 1. There were no statistically significant differences in age, sex, diseases, drug species, preoperative BCVA, preoperative IOP, or postoperative IOP between the ineffective and effective groups $(p>0.05)$. Moreover, there were no statistically significant differences between the groups with respect to level of white blood cells, neutrophils, monocytes, or lymphocytes $(p>0.05)$. 
Table 2. Comparison of the ineffective group and the effective group with BRVO-ME

\begin{tabular}{|c|c|c|c|}
\hline & $\begin{array}{l}\text { Ineffective group } \\
(n=103)\end{array}$ & $\begin{array}{l}\text { Effective group } \\
(n=99)\end{array}$ & $p$ value \\
\hline \multicolumn{4}{|l|}{ Baseline characteristics } \\
\hline Age, years & $59.97 \pm 10.38$ & $61.11 \pm 11.11$ & 0.32 \\
\hline Male/female & $42 / 61$ & $41 / 58$ & 0.97 \\
\hline Ranibizumab/conbercept & $73 / 30$ & $63 / 36$ & 0.27 \\
\hline Preoperative BCVA, logMAR & $0.70 \pm 0.39$ & $0.72 \pm 0.36$ & 0.60 \\
\hline Postoperative BCVA, logMAR & $0.72 \pm 0.41$ & $0.48 \pm 0.28$ & $<0.01$ \\
\hline Changes in BCVA, logMAR & $0.02 \pm 0.07$ & $0.26 \pm 0.18$ & $<0.01$ \\
\hline Preoperative IOP, mm Hg & $14.31 \pm 2.42$ & $14.00 \pm 2.92$ & 0.51 \\
\hline Postoperative IOP, $\mathrm{mm} \mathrm{Hg}$ & $13.76 \pm 2.67$ & $13.76 \pm 2.73$ & 0.97 \\
\hline Changes in IOP, $\mathrm{mm} \mathrm{Hg}$ & $2.55 \pm 1.63$ & $2.32 \pm 1.52$ & 0.38 \\
\hline \multicolumn{4}{|l|}{ Laboratory findings } \\
\hline White blood cells, $\times 10^{9} / \mathrm{L}$ & $5.38 \pm 1.53$ & $5.53 \pm 1.25$ & 0.23 \\
\hline Neutrophils, $\times 10^{9} / \mathrm{L}$ & $3.05 \pm 1.24$ & $3.23 \pm 0.86$ & 0.01 \\
\hline Monocytes, $\times 10^{9} / \mathrm{L}$ & $0.40 \pm 0.17$ & $0.38 \pm 0.14$ & 0.44 \\
\hline Platelets, $\times 10^{9} / \mathrm{L}$ & $185.94 \pm 51.47$ & $216.47 \pm 53.04$ & $<0.01$ \\
\hline Lymphocytes, $\times 10^{9} / \mathrm{L}$ & $1.77 \pm 0.47$ & $1.75 \pm 0.57$ & 0.54 \\
\hline NLR & $1.82 \pm 0.84$ & $2.01 \pm 0.80$ & 0.02 \\
\hline MLR & $0.23 \pm 0.11$ & $0.24 \pm 0.10$ & 0.72 \\
\hline PLR & $108.46 \pm 28.74$ & $132.14 \pm 42.34$ & $<0.01$ \\
\hline
\end{tabular}

BRVO, branch retinal vein occlusion; ME, macular edema; BCVA, best-corrected visual acuity; IOP, intraocular pressure; NLR, neutrophil-to-lymphocyte ratio; MLR, monocyte-to-lymphocyte ratio; PLR, plateletto-lymphocyte ratio.

Change in BCVA was regarded as the standard grouping of the patients, and this parameter showed significant differences between the ineffective and effective groups $(0.02 \pm 0.08$ vs. $0.26 \pm 0.19, p<0.05)$; however, the magnitude of changes in intraocular pressure was not significantly different between the groups $(2.47 \pm 1.68$ vs. 2.13 $\pm 1.47, p>0.05)$. Platelet levels were higher in the effective group than in the ineffective group $(221.87 \pm 58.59$ vs. $189.51 \pm 54.78, p<0.01)$. A high PLR was correlated with a better prognosis of effective treatment $(138.03 \pm 48.61$ for the effective group; $106.79 \pm 27.28$ for the ineffective group, $p<0.01)$. The mean NLR values were $1.92 \pm 0.89$ and $2.20 \pm 1.40$ in the ineffective and effective groups, respectively, and the difference between the 2 groups was statistically significant $(p=0.01, p<0.05)$. The mean MLR values were $0.24 \pm 0.11$ and $0.26 \pm 0.13$ in the ineffective and effective groups, respectively; the difference between the 2 groups was not statistically significant $(p>0.05)$.

Regarding the subtypes of RVO-ME, including BRVO and CRVO (i.e., BRVO-ME and CRVO-ME), there were no statistically significant differences in age, sex, drug treatment, preoperative BCVA, preoperative IOP, postoperative IOP, or changes in IOP between the ineffective and effective groups ( $p>0.05$; Tables 2,3$)$. Similarly, there were no significant differences in levels of white blood cells, neutrophils, monocytes, and lymphocytes between the ineffective and effective groups $(p>0.05)$. Platelet count was a significant factor, and was higher in the effective group than in the ineffective group (216.47 \pm 53.04 vs. $185.94 \pm 51.47$ for BRVO-ME; $231.07 \pm 66.05$ vs. $196.20 \pm 60.44$ for CRVO-ME, $p<0.05$ ). The mean NLR value was significantly different in patients with BRVO-ME (1.82 \pm 0.84 for the ineffective group; $2.01 \pm$ 0.80 for the effective group, $p<0.05$ ), but not in patients with CRVO-ME $(2.12 \pm 0.95$ for the ineffective group; $2.51 \pm 2.02$ for the effective group, $p>0.05$ ). There was also a statistically significant difference in mean PLR values between the effective and ineffective groups for both RVO-ME subtypes (132.14 \pm 42.34 vs. $108.46 \pm 28.74$ for BRVO-ME; $148.07 \pm 56.76$ vs. $103.66 \pm 24.24$ for CRVO$\mathrm{ME}, p<0.05)$. However, the mean MLR was not significantly different between the 2 groups $(p>0.05)$.

In RVO-ME and its subtypes, the levels of white blood cells, neutrophils, monocytes, and lymphocytes did not significantly differ between the ranibizumab and conbercept groups ( $p>0.05$; Table 4$)$. Although the mean MLR 
Table 3. Comparison of the ineffective group and the effective group with CRVO-ME

\begin{tabular}{|c|c|c|c|}
\hline & $\begin{array}{l}\text { Ineffective group } \\
(n=55)\end{array}$ & $\begin{array}{l}\text { Effective group } \\
(n=58)\end{array}$ & $p$ value \\
\hline \multicolumn{4}{|l|}{ Baseline characteristics } \\
\hline Age, years & $52.40 \pm 13.00$ & $55.14 \pm 13.12$ & 0.27 \\
\hline Male/female & $37 / 18$ & $33 / 25$ & 0.25 \\
\hline Ranibizumab/conbercept & $32 / 23$ & $37 / 21$ & 0.54 \\
\hline Preoperative BCVA, logMAR & $0.86 \pm 0.40$ & $0.92 \pm 0.42$ & 0.43 \\
\hline Postoperative BCVA, logMAR & $0.86 \pm 0.44$ & $0.67 \pm 0.33$ & 0.02 \\
\hline Changes in BCVA, logMAR & $0.04 \pm 0.10$ & $0.25 \pm 0.19$ & $<0.01$ \\
\hline Preoperative IOP, mm Hg & $13.73 \pm 2.89$ & $14.12 \pm 2.90$ & 0.52 \\
\hline Postoperative IOP, $\mathrm{mm} \mathrm{Hg}$ & $13.95 \pm 2.73$ & $13.66 \pm 2.67$ & 0.57 \\
\hline Changes in IOP, $\mathrm{mm} \mathrm{Hg}$ & $2.33 \pm 1.76$ & $1.81 \pm 1.30$ & 0.17 \\
\hline \multicolumn{4}{|l|}{ Laboratory findings } \\
\hline White blood cells, $\times 10^{9} / \mathrm{L}$ & $6.57 \pm 2.38$ & $6.05 \pm 1.82$ & 0.28 \\
\hline Neutrophils, $\times 10^{9} / \mathrm{L}$ & $3.91 \pm 1.87$ & $3.73 \pm 1.54$ & 0.61 \\
\hline Monocytes, $\times 10^{9} / \mathrm{L}$ & $0.47 \pm 0.18$ & $0.45 \pm 0.15$ & 0.65 \\
\hline Platelets, $\times 10^{9} / \mathrm{L}$ & $196.20 \pm 60.44$ & $231.07 \pm 66.05$ & $<0.01$ \\
\hline Lymphocytes, $\times 10^{9} / \mathrm{L}$ & $1.94 \pm 0.65$ & $1.69 \pm 0.59$ & 0.03 \\
\hline NLR & $2.12 \pm 0.95$ & $2.51 \pm 2.02$ & 0.27 \\
\hline MLR & $0.26 \pm 0.12$ & $0.30 \pm 0.16$ & 0.11 \\
\hline PLR & $103.66 \pm 24.24$ & $148.07 \pm 56.76$ & $<0.01$ \\
\hline
\end{tabular}

CRVO, central retinal vein occlusion; ME, macular edema; BCVA, best-corrected visual acuity; IOP, intraocular pressure; NLR, neutrophil-to-lymphocyte ratio; MLR, monocyte-to-lymphocyte ratio; PLR, plateletto-lymphocyte ratio.

Table 4. Comparison of ME secondary to RVO and its subtypes in the effective group

\begin{tabular}{|c|c|c|c|c|c|c|c|c|c|}
\hline & \multicolumn{3}{|l|}{ RVO group } & \multicolumn{3}{|l|}{ BRVO group } & \multicolumn{3}{|l|}{ CRVO group } \\
\hline Male/female & $49 / 51$ & $27 / 30$ & 0.84 & $28 / 35$ & $15 / 21$ & 0.79 & $21 / 16$ & $12 / 9$ & 0.98 \\
\hline White blood cells, $\times 10^{9} / \mathrm{L}$ & $5.84 \pm 1.56$ & $5.52 \pm 1.38$ & 0.20 & $5.63 \pm 1.19$ & $5.35 \pm 1.35$ & 0.10 & $6.17 \pm 2.01$ & $5.81 \pm 1.43$ & 0.48 \\
\hline Neutrophils, $\times 10^{9} / \mathrm{L}$ & $3.47 \pm 1.25$ & $3.32 \pm 1.05$ & 0.52 & $3.29 \pm 0.84$ & $3.12 \pm 0.89$ & 0.24 & $3.77 \pm 1.71$ & $3.67 \pm 1.22$ & 0.80 \\
\hline Lymphocytes, $\times 10^{9} / \mathrm{L}$ & $1.77 \pm 0.55$ & $1.66 \pm 0.62$ & 0.10 & $1.78 \pm 0.52$ & $1.68 \pm 0.66$ & 0.14 & $1.73 \pm 0.62$ & $1.62 \pm 0.56$ & 0.41 \\
\hline NLR & $2.21 \pm 1.63$ & $2.18 \pm 0.85$ & 0.31 & $1.99 \pm 0.79$ & $2.05 \pm 0.83$ & 0.91 & $2.58 \pm 2.46$ & $2.40 \pm 0.85$ & 0.10 \\
\hline MLR & $0.25 \pm 0.14$ & $0.27 \pm 0.11$ & 0.045 & $0.22 \pm 0.89$ & $0.26 \pm 0.12$ & 0.076 & $0.30 \pm 0.18$ & $0.29 \pm 0.10$ & 0.33 \\
\hline PLR & $135.96 \pm 50.01$ & $141.66 \pm 46.26$ & 0.34 & $130.21 \pm 38.54$ & $135.53 \pm 48.69$ & 0.96 & $145.74 \pm 64.52$ & $152.17 \pm 40.73$ & 0.15 \\
\hline
\end{tabular}

RVO, retinal vein occlusion; ME, macular edema; BRVO, branch RVO; CRVO, central RVO; BCVA, best-corrected visual acuity; IOP, intraocular pressure; NLR, neutrophil-to-lymphocyte ratio; MLR, monocyte-to-lymphocyte ratio; PLR, platelet-to-lymphocyte ratio.

value was significantly higher in the conbercept group than in the ranibizumab group among all patients $(0.27 \pm$ 0.11 vs. $0.25 \pm 0.14, p<0.05$ ), this difference was not statistically significant in patients with $\mathrm{BRVO}-\mathrm{ME}$ or in patients with CRVO-ME. In addition, the other 2 inflam- matory predictors (NLR and PLR) did not significantly differ between the 2 treatment groups $(p>0.05)$.

ROC curve analysis showed that the optimal cutoff value of PLR for predicting an effective outcome was 97.92, with $81.5 \%$ sensitivity and $44.3 \%$ specificity (AUC 0.70 ; 
Table 5. Comparison of ROC curves for NLR, PLR, and platelets in the prediction of effective treatment

\begin{tabular}{lllll}
\hline & AUC & $95 \%$ CI & Sensitivity, \% & Specificity, \% \\
\hline NLR $(>1.98)$ & 0.58 & $0.518-0.643$ & 50.3 & 63.3 \\
PLR $(>97.92)$ & 0.70 & $0.643-0.756$ & 81.5 & 44.3 \\
Platelet count $\left(>178.5 \times 10^{9} / \mathrm{L}\right)$ & 0.65 & $0.593-0.713$ & 81.5 & 42.4 \\
\hline
\end{tabular}

NLR, neutrophil-to-lymphocyte ratio; PLR, platelet-to-lymphocyte ratio; ROC, receiver operating characteristic; AUC, area under the curve; $\mathrm{CI}$, confidence interval.

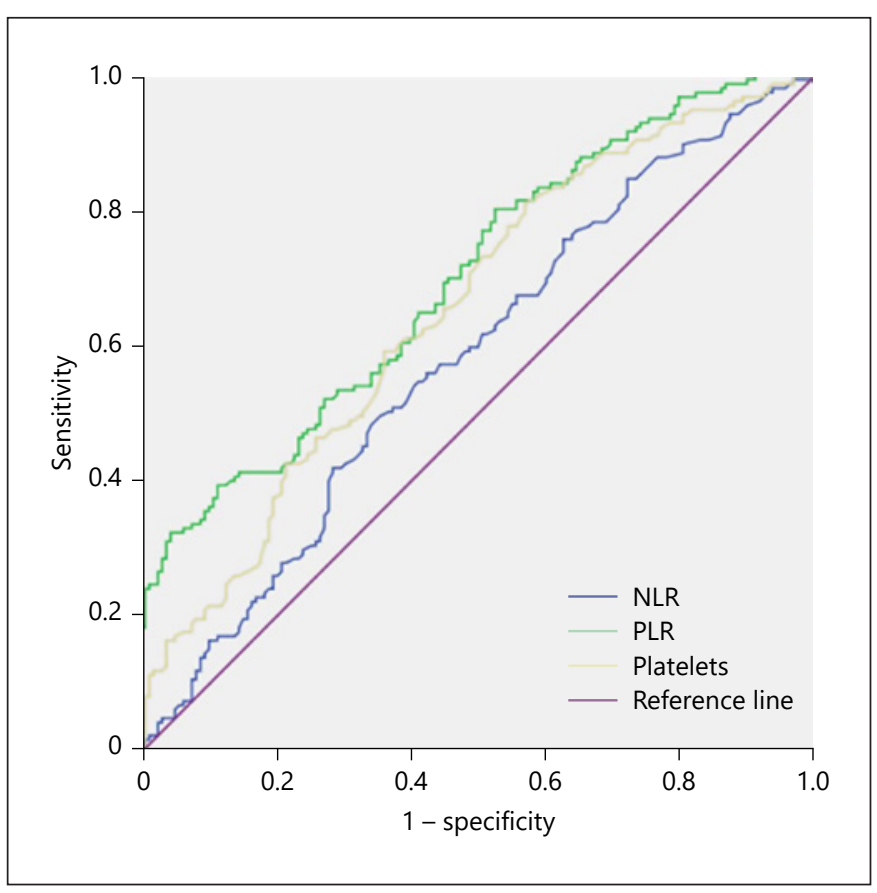

Fig. 1. Comparison of ROC curves for NLR, PLR, and platelets in the prediction of effective treatment.

95\% CI 0.643-0.756), Furthermore, the optimal cutoff value of NLR was 1.98 , with $50.3 \%$ sensitivity and $63.3 \%$ specificity (AUC 0.58 ; 95\% CI 0.518-0.643). The optimal cutoff value of platelets was 178.5 , with $81.5 \%$ sensitivity and $42.4 \%$ specificity (AUC 0.65 ; 95\% CI $0.593-0.713$; Table 5; Fig. 1). The areas under the ROC curves of each of the 3 variables were compared.

\section{Discussion}

The present study showed that pretreatment platelets, PLR, and NLR were significantly associated with an effective visual outcome in patients with RVO-ME and its subtypes following anti-VEGF treatment. Furthermore, compared with NLR and platelets, higher PLR may be a stronger and better predictor for anti-VEGF treatment. Notably, the level of MLR was related with the efficacy of ranibizumab or conbercept treatment among the broader subset of patients with RVO-ME, but this association was lost in the subgroup analysis.

As a common retinal vascular pathology, RVO is intractable after the development of ME or neovascular glaucoma, especially in older patients. ME is the most common cause of visual impairment and is always accompanied by high vascular permeability and an increased level of VEGF [14]. Although it can be easily diagnosed, the pathogenesis of RVO-ME is complicated and remains poorly understood. Systemic diseases such as hypertension and diabetes mellitus, as well as high homocysteine and thrombophilia diseases, are associated with RVO-ME $[15,16]$. Moreover, intravitreal injection treatments targeting the reduction of inflammatory factors, especially VEGF, could increase visual acuity in patients with RVO-ME, suggesting that inflammation is a key aspect in the pathogenesis of RVO-ME [9].

White blood cells and their subtypes play crucial roles in modulating inflammation reflection in cardiovascular disease [17]. Highly activated neutrophils release an extensive mixture of chemical mediators, including VEGF, reactive oxygen species, and cytokines; these can impair the endothelial barrier, thereby increasing vascular permeability and aggravating ME [18]. In contrast, low levels of lymphocytes are associated with a poor prognosis in patients with coronary artery disease [19]. The NLR, which is calculated based on the neutrophil and lymphocyte count, is associated with inflammation and has been widely used as a marker to predict systemic inflammatory responses and outcomes. Previous studies have shown the prognostic value of NLR with respect to mortality in cardiovascular diseases [20], malignancies [21], and cerebrovascular disease [22]. In the present study we found that the pretreatment NLR was associated with treatment outcomes in patients with RVO-ME who received antiVEGF therapy; high NLR (>1.98) was more likely to re- 
sult in better visual acuity, suggesting effective treatment. Dursun et al. [13] demonstrated that higher NLR (>1.89) was associated with the progress of RVO and could be used as a tool for the prediction of systemic inflammation in patients with RVO. Therefore, NLR could be a significant prognostic factor for RVO-ME patients. However, among RVO subtypes, we found that NLR was related to the treatment outcome for patients with BRVO-ME, but not for patients with CRVO-ME. In contrast, Turkseven Kumral et al. [23] showed that NLR was not affected in patients with BRVO. Because of these conflicting results, it remains unclear whether NLR would be a significant prognostic factor for patients with ME secondary to RVO subtypes. The results in this study may have been influenced by the limitation of the study design (described later in this section); therefore, we will further explore this relationship in future studies.

In addition to their physiological or pathological functions in thrombosis and hemostasis, platelets can play an important role in inflammation and release of VEGF [24, 25]. Xu et al. [26] showed that platelets can serve as effectors of inflammation injury in a variety of pulmonary disorders and syndromes. In addition, Vinholt et al. [27] found that the platelet count could be useful for stratification of cardiovascular risk - a high platelet count was associated with an increased risk of cardiovascular disease. We found that the pretreatment platelet count was associated with outcomes in patients with RVO-ME and its subtypes when patients were treated with anti-VEGF drugs, and that a high platelet count $(>178.5)$ was predictive of better postoperative BCVA outcomes. Furthermore, previous studies showed that the PLR were closely related to the progression and prognosis of cancers [28] and venous thrombosis [25]. Our results indicated that high PLR, which combines the predictive value of platelets and lymphocytes, could be a better prognostic factor for RVO-ME and its subtypes in patients treated with anti-VEGF drugs when platelet count was assessed based on ROC curve analysis. However, the potential mechanism underlying the association between elevated PLR and better outcomes is poorly understood. Seropian et al. [29] reported that higher PLR was positively correlated with cardiovascular diseases. In addition, some studies have shown relationships between RVO and various thrombotic diseases [30] and systemic inflammatory diseases [31]. Therefore, PLR may be a significant prognostic factor for RVO-ME and its subtypes in patients treated with anti-VEGF drugs.

The MLR is another inflammatory factor that is closely associated with progressive cardiovascular disease [32] and retinal illness [33]. In this study, investigation of the relationship between IVR or IVC outcomes and MLR showed that MLR was associated with the efficacy of IVR or IVC treatment of patients with RVO-ME, which may be due to the pharmacological mechanisms underlying treatment with ranibizumab and conbercept. Ranibizumab is an anti-VEGF-A monoclonal antibody fragment that does not contain the Fc antibody region [34], whereas conbercept is a novel recombinant fusion protein that contains the $\mathrm{Fc}$ antibody region, which binds the second, third, and fourth Ig domains of the VEGF receptor [35]. The 2 anti-VEGF drugs prevent the combination between VEGF and receptor in different ways. However, our results indicated that the mean MLR value could not predict the outcome of treatment efficacy for RVO-ME and its subtypes' efficient group. Li et al. [36] suggested that IVR or IVC did not cause an obvious difference in the treatment in BRVO-ME. Thus, the MLR may be related to the prognosis of patients with RVO-ME who were treated with anti-VEGF in the effective group, but this relationship was not confirmed in the present study. Further studies are therefore required to reach a definitive conclusion.

We are aware of the limitations of this study. Firstly, changes in BCVA were used to judge whether the treatment was effective, which may have caused reporting bias. Next, other potential markers of inflammatory status (e.g., C-reactive protein and interleukin) and their associations with PLR, NLR, and MLR were not investigated. Lastly, the retrospective design and low patient number may limit the generalizability of our conclusions. Because of the convenience and low cost of the parameter, there may be value in determining whether PLR is predictive or prognostic in patients with RVO$\mathrm{ME}$ who are receiving anti-VEGF drugs. Consequently, a high pretreatment PLR may be strongly correlated with effective visual improvement prognosis in patients with RVO-ME and its subtypes who are treated with antiVEGF drugs. Further studies are needed to confirm the predictive and prognostic value of PLR for outcomes in patients with RVO-ME and its subtypes after anti-VEGF treatment.

\section{Statement of Ethics}

This study was performed in accordance with the principles of the Declaration of Helsinki and was approved by the Institutional Review Board of the First Affiliated Hospital of Nanchang University. All of the subjects signed informed consent forms. 


\section{Conflict of Interest Statement}

The datasets used and analyzed during the current study are available from the corresponding author on reasonable request. The authors declare that they have no competing interests.

\section{Funding Sources}

This study was supported by the National Natural Science Foundation of China (No. 81760179, No. 81360151), Natural Science Foundation of Jiangxi Province (No. 20171BAB205046), Key Research Plan of Jiangxi Provincial Department of Science and Technology (No.20192BBG70042), Key Foundation of Education Department of Jiangxi Province (No. GJJ160033), Health Development Planning Commission Science Foundation of Jiangxi Prov- ince (No. 20185118, No. 20141031), Technology and Science Foundation of Jiangxi Province (No. 20141BBG70027), Health and Family Planning Commission Traditional Chinese Medicine Foundation of Jiangxi Province (No. 20185118), and Basic Health Appropriate Technology Spark Promotion Program of Jiangxi Province (No. 20188007). The funders had no role in the design of the study, in the collection, analysis, and interpretation of data, or in writing the manuscript.

\section{Author Contributions}

X.W.: conceived and designed the experiments. J.R.: wrote the manuscript. N.W., X.Q., and Y.H.: perform the data analyses. Y.W., Y.C., L.Y., and H.H.: collect data and information. S.L. and L.S.: helped perform the analysis and follow-up patients.

\section{References}

1 Jonas JB, Monés J, Glacet-Bernard A, Coscas G. Retinal vein occlusions. Dev Ophthalmol. 2017;58:139-67.

2 Rogers S, McIntosh RL, Cheung N, Lim L, Wang JJ, Mitchell P, et al.; International Eye Disease Consortium. The prevalence of retinal vein occlusion: pooled data from population studies from the United States, Europe, Asia, and Australia. Ophthalmology. 2010 Feb;117(2):313-9.e1.

3 Zhao F, Du F, Shi D, Zhou W, Jiang Y, Ma L. Mapping research trends of retinal vein occlusion from 2009 to 2018: a bibliometric analysis. PeerJ. 2019 Aug;7:e7603.

4 Babiuch AS, Han M, Conti FF, Wai K, Silva FQ, Singh RP. Association of disorganization of retinal inner layers with visual acuity response to anti-vascular endothelial growth factor therapy for macular edema secondary to retinal vein occlusion. JAMA Ophthalmol. 2019 Jan;137(1):38-46.

5 Noma H, Mimura T, Eguchi S. Association of inflammatory factors with macular edema in branch retinal vein occlusion. JAMA Ophthalmol. $2013 \mathrm{Feb} ; 131(2): 160-5$

6 Rezar-Dreindl S, Eibenberger K, Pollreisz A, Bühl W, Georgopoulos M, Krall C, et al. Effect of intravitreal dexamethasone implant on intra-ocular cytokines and chemokines in eyes with retinal vein occlusion. Acta Ophthalmol. 2017 Mar;95(2):e119-27.

7 Vinores SA, Derevjanik NL, Ozaki H, Okamoto N, Campochiaro PA. Cellular mechanisms of blood-retinal barrier dysfunction in macular edema. Doc Ophthalmol. 1999;97(34):217-28.

8 Noma H, Funatsu H, Yamasaki M, Tsukamoto $\mathrm{H}$, Mimura T, Sone T, et al. Pathogenesis of macular edema with branch retinal vein occlusion and intraocular levels of vascular endothelial growth factor and interleukin-6. Am J Ophthalmol. 2005 Aug;140(2):256-61.
9 Jiang Y, Mieler WF. Update on the use of anti-VEGF intravitreal therapies for retinal vein occlusions. Asia Pac J Ophthalmol. 2017 NovDec;6(6):546-53.

10 Krakowska M, Dębska-Szmich S, Czyżykowski R, Zadrożna-Nowak A, Potemski P. The prognostic impact of neutrophil-to-lymphocyte ratio, lymphocyte-to-monocyte ratio, and platelet-to-lymphocyte ratio in patients with advanced colorectal cancer treated with first-line chemotherapy. Prz Gastroenterol. 2018;13(3):218-22.

11 Bakirci EM, Topcu S, Kalkan K, Tanboga IH, Borekci A, Sevimli S, et al. The role of the nonspecific inflammatory markers in determining the anatomic extent of venous thromboembolism. Clin Appl Thromb Hemost. 2015 Mar;21(2):181-5

$12 \mathrm{Fu} \mathrm{H}$, Qin B, Hu Z, Ma N, Yang M, Wei T, et al. Neutrophil- and platelet-to-lymphocyte ratios are correlated with disease activity in rheumatoid arthritis. Clin Lab. 2015;61(3-4): 269-73.

13 Dursun A, Ozturk S, Yucel H, Ozec AV, Dursun FG, Toker MI, et al. Association of neutrophil/lymphocyte ratio and retinal vein occlusion. Eur J Ophthalmol. 2015 Jul-Aug; 25(4):343-6.

14 Rehak M, Wiedemann P. Retinal vein thrombosis: pathogenesis and management. J Thromb Haemost. 2010 Sep;8(9):1886-94.

15 Jaulim A, Ahmed B, Khanam T, Chatziralli IP. Branch retinal vein occlusion: epidemiology, pathogenesis, risk factors, clinical features, diagnosis, and complications. An update of the literature. Retina. 2013 May;33(5): 901-10.

16 Kesler A, Shalev V, Rogowski O, Shimron O, Shainberg B, Sela BA, et al. Comparative analysis of homocysteine concentrations in patients with retinal vein occlusion versus thrombotic and atherosclerotic disorders. Blood Coagul Fibrinolysis. 2008 Jun;19(4): $259-62$.
17 Horne BD, Anderson JL, John JM, Weaver A, Bair TL, Jensen KR, et al.; Intermountain Heart Collaborative Study Group. Which white blood cell subtypes predict increased cardiovascular risk? J Am Coll Cardiol. 2005 May;45(10):1638-43.

18 Rodrigues SF, Granger DN. Blood cells and endothelial barrier function. Tissue Barriers. 2015 Apr;3(1-2):e978720.

19 Bian C, Wu Y, Shi Y, Xu G, Wang J, Xiang M, et al. Predictive value of the relative lymphocyte count in coronary heart disease. Heart Vessels. 2010 Nov;25(6):469-73.

20 González-Fajardo JA, Brizuela-Sanz JA, Aguirre-Gervás B, Merino-Díaz B, Del Río-Solá L, Martín-Pedrosa M, et al. Prognostic significance of an elevated neutrophil-lymphocyte ratio in the amputation-free survival of patients with chronic critical limb ischemia. Ann Vasc Surg. 2014 May;28(4):999-1004.

21 Bagley SJ, Kothari S, Aggarwal C, Bauml JM, Alley EW, Evans TL, et al. Pretreatment neutrophil-to-lymphocyte ratio as a marker of outcomes in nivolumab-treated patients with advanced non-small-cell lung cancer. Lung Cancer. 2017 Apr;106:1-7.

22 Lattanzi S, Cagnetti C, Provinciali L, Silvestrini M. Neutrophil-to-lymphocyte ratio predicts the outcome of acute intracerebral hemorrhage. Stroke. 2016 Jun;47(6):1654-7.

23 Turkseven Kumral E, Yenerel NM, Ercalik NY, Imamoglu S, Vural ET. Neutrophil/lymphocyte ratio and mean platelet volume in branch retinal vein occlusion. Saudi J Ophthalmol. 2016 Apr-Jun;30(2):105-8.

24 Ding D, Liu X, Duan J, Guo SW. Platelets are an unindicted culprit in the development of endometriosis: clinical and experimental evidence. Hum Reprod. 2015 Apr;30(4):812-32.

25 Franco AT, Corken A, Ware J. Platelets at the interface of thrombosis, inflammation, and cancer. Blood. 2015 Jul;126(5):582-8. 
26 Xu XR, Zhang D, Oswald BE, Carrim N, Wang X, Hou Y, et al. Platelets are versatile cells: new discoveries in hemostasis, thrombosis, immune responses, tumor metastasis and beyond. Crit Rev Clin Lab Sci. 2016 Dec; 53(6):409-30.

27 Vinholt PJ, Hvas AM, Frederiksen H, Bathum L, Jørgensen MK, Nybo M. Platelet count is associated with cardiovascular disease, cancer and mortality: A population-based cohort study. Thromb Res. 2016 Dec;148:136-42.

28 Bailon-Cuadrado M, Choolani-Bhojwani E, Tejero-Pintor FJ, Sanchez-Gonzalez J, Rodriguez-Lopez M, Perez-Saborido B, et al. Preoperative platelet-lymphocyte ratio is an independent factor of poor prognosis after curative surgery for colon cancer. Updates Surg. 2018 Mar;70(1):33-9.
29 Seropian IM, Romeo FJ, Pizarro R, Vulcano NO, Posatini RA, Marenchino RG, et al. Neutrophil-to-lymphocyte ratio and platelet-tolymphocyte ratio as predictors of survival after heart transplantation. ESC Heart Fail. 2018 Feb;5(1):149-56.

30 Janssen MC, den Heijer M, Cruysberg JR, Wollersheim H, Bredie SJ. Retinal vein occlusion: a form of venous thrombosis or a complication of atherosclerosis? A meta-analysis of thrombophilic factors. Thromb Haemost. 2005 Jun;93(6): 1021-6.

31 El FL, Khaldi N, Hmaied W, Ksantini I, Mestiri A, Doggi M. [Retinal Venous Occlusion in Behçet Disease]. Rev Med Interne. 2007 Nov; 28(11):742-5. French.

32 Serföző G, Horváth T, Földesi I, Rafael B, von Känel R, Keresztes M. The monocyte-to-lymphocyte ratio correlates with psycho-neuroinflammatory factors in patients with stable coronary artery disease. Neuroimmunomodulation. 2016;23(2):67-74.
$33 \mathrm{Hu}$ YX, Xu XX, Shao Y, Yuan GL, Mei F, Zhou $\mathrm{Q}$, et al. The prognostic value of lymphocyteto-monocyte ratio in retinopathy of prematurity. Int J Ophthalmol. 2017 Nov; 10(11): 1716-21.

34 Avery RL, Castellarin AA, Steinle NC, Dhoot DS, Pieramici DJ, See R, et al. Systemic pharmacokinetics and pharmacodynamics of intravitreal aflibercept, bevacizumab, and ranibizumab. Retina. 2017 Oct;37(10):1847-58.

35 Zhang M, Yu D, Yang C, Xia Q, Li W, Liu B, et al. The pharmacology study of a new recombinant human VEGF receptor-fc fusion protein on experimental choroidal neovascularization. Pharm Res. 2009 Jan;26(1):204-10.

36 Li F, Sun M, Guo J, Ma A, Zhao B. Comparison of conbercept with ranibizumab for the treatment of macular edema secondary to branch retinal vein occlusion. Curr Eye Res. 2017 Aug;42(8):1174-8. 